\title{
Analysis of Shielded Twisted Pair Cable to External Field Coupling by Expanded Chain Matrix Modeling
}

\author{
Yong-Sun Cho*, Hyun-Kyo Jung*, Changyul Cheon** and Young-Seek Chung ${ }^{\dagger}$
}

\begin{abstract}
In this paper, a numerical method for analyzing coupling between high-altitude electromagnetic pulse (HEMP) as external field and a shielded twisted pair (STP) cable is proposed, which is based on an expanded chain matrix. Load responses of electromagnetic (EM) field excitation in uniform transmission line (TL) are solved by Baum-Liu-Tesche (BLT) equations in frequency domain, however, it is difficult to apply BLT equations to solve load responses of STP cable because the iteratively changing configuration of each twisted pairs are involved in cable. To avoid this problem and decrease memory and CPU time, we proposed the expanded chain matrix modeling method that is calculated using ABCD parameters, and applied multi-conductor transmission line (MTL) theory to consider the EMP coupling effectiveness of each twisted pairs. The results implemented by the proposed method are presented and compared with those obtained by the finitedifference time domain (FDTD) method as a kind of 3D full wave analysis.
\end{abstract}

Keywords: Baum-Liu-Tesche (BLT), Chain matrix, Coupling, Electromagnetic (EM), Finitedifference Time Domain (FDTD), High-altitude Electromagnetic Pulse (HEMP), Multi-conductor Transmission Line (MTL), Shielded Twisted Pair (STP), Transmission Line (TL).

\section{Introduction}

The STP cables are frequently used to transmit the signal between equipment contained within two protective enclosures to reduce electromagnetic interference (EMI) [1]. Nevertheless, the transient EM disturbance produced by external fields generating EMI can easily couple the core cables, and the accuracy of the data acquisition will affect to point of entry (POE) results in a shield that is not perfect $[2,3]$. Therefore, it is important to analyze the induced responses in the STP cable to predict performances of the cable against external EMP in terms of shielding effectiveness. In other words, the effects of HEMP-induced electrical transients on electronic equipment are important in the survivability assessment of electric power systems [4]. A first step in this process is the determination of the cable currents and voltages that can couple energy into the equipment that might be subject to failure. This paper considers one particular system and illustrates the techniques used for estimating the response of the system to HEMP. So, the system response to HEMP which is a transient plane electromagnetic wave produced by a high-altitude nuclear explosion to the STP cable can be estimated.

Traditionally, the behavior of the induced responses on

$\doteqdot$ Corresponding Author: Dept. of Electronics Convergence Engineering, Kwangwoon University, Korea. (yschung@kw.ac.kr)

* Dept. of Electrical \& Computer Engineering, Seoul National University, Korea. (raphaelz@snu.ac.kr, hkjung@snu.ac.kr)

** Dept. of Electrical \& Computer Engineering, University of Seoul, Korea. (changyul@uos.ac.kr)

Received: February 13, 2014; Accepted: July 1, 2014 the inner conductors can be evaluated using MTL theory [5]. For complex structure of the cable layout such as twisted pair or in a high frequency range, a full wave analysis such as the FDTD method is a very popular way to analyze transient fields in 3-dimensional domains. With the development of computing technology, a significant research effort has been made to address the development of the FDTD method to analyze the wire structure with a size less than the entire cell dimension [6]-[8]. However, it requires significant computer memory storage and $\mathrm{CPU}$ time [9].

Meanwhile, for describing the coupling of an external EM field to a cable using the TL theory, BLT equations have been studied in cable problems. Further, in case of nconductors of finite length which are referred to as MTL, BLT equations that are generalized into a super-matrix can be applied to solve load responses. However, there is a limit to the use of BLT equations to analyze HEMP coupling with a STP cable because of iteratively changing configuration of each twisted pairs in the whole structure. It means that conventional BLT equations do not consider repeatedly changing components of the per-unit-length (PUL) parameters [5].

In this paper, to overcome the mentioned problems and limitations, a novel method based on the expanded chain matrix is presented using MTL theory as extended version of analysis of field coupling with unshielded twisted pair cable [5]. The proposed method uses ABCD parameters, which consider the coupling component of each line, and the twisted pairs are modeled by finite number of small sections. For analyzing the external HEMP coupling effect 


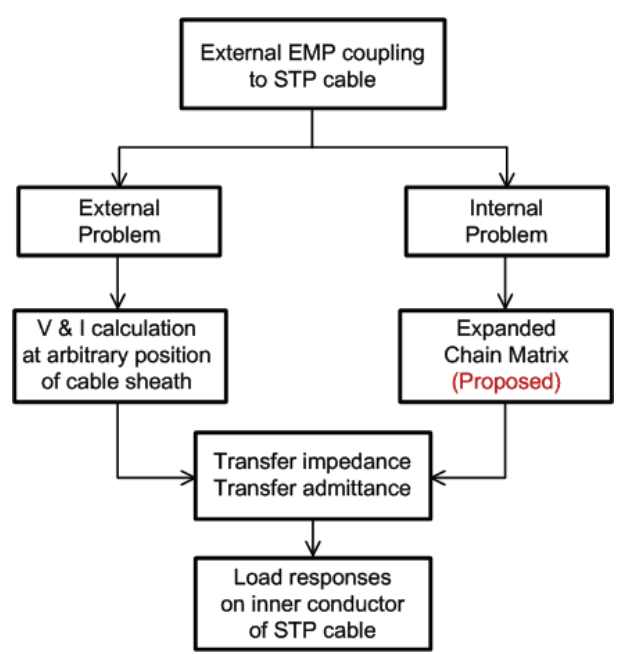

Fig. 1. The concept of entire problem.

in an STP cable, we first calculated both a shield current and a shield to ground voltage induced by an external HEMP as an external problem. Then, the behavior of the induced response on the inner conductors of the STP cable can be obtained by combining transfer impedance and transfer admittance terms with proposed modeling method.

The paper is organized as follows: In Section II, the concept of the entire problem is presented. The detailed mathematical models of external and internal problems are outlined in Section III, some results are obtained, with different lengths, heights, and ground conductivities. Then, the FDTD method is used to demonstrate the accuracy of the proposed method in Section IV. In Section V, we present some conclusions.

\section{Concept of Problem}

As shown in Fig. 1, for analyzing the HEMP coupling with STP cable, the concept of the entire problem needs to be divided into two parts: external and internal, because it has unwanted response at the "shielding" equipment. The external problem stands for the external HEMP coupling with TL that is modeled by the cable sheath and the ground plane, which is sensitive to the external EM field environment because of its direct exposure. General solutions for the voltage and current responses at an arbitrary point of a finite cable sheath can be derived by [10]. In addition, the responses on the cable sheath are equivalent to excitation sources through transfer impedance and transfer admittance, thus it can be seen that the external and the internal problem are linked together. Although the internal core wires are less affected by external HEMP, the portions of the external electric and magnetic fields are able to penetrate through imperfections in the cable sheath, and these induce internal current and voltage responses. The behavior of the induced responses of the inner conductors of a STP cable, as a kind of internal problem, can be obtained by combining the expanded chain matrix proposed and concept of transfer impedance and transfer admittance terms.

\section{Mathematical Model}

In its simplest configuration, a STP cable consists of a cable with the sheath connected to each enclosure. As shown in Fig. 2, the enclosures and the STP cable are usually located over a ground plane with a height $h$ and length is $L$. The two enclosures are connected to the ground plane by external impedances $Z_{l, 2 \text { out }}$.

\subsection{External problem}

A time-varying external EM field will induce both a shield current and a shield to ground voltage. This can be thought of as arising from a set of distributed voltages and currents located along the cable sheath as depicted in Fig. 3. The distributed voltage and current sources terms can be calculated with

$$
\begin{gathered}
V_{s}(x)=-j \omega \mu \int_{0}^{h} H_{y}^{e x}(x, z) d z \\
I_{s}(x)=-Y^{\prime} \int_{0}^{h} E_{z}^{e x}(x, z) d z
\end{gathered}
$$

where $\omega$ is angular frequency, $\mu$ is the permeability of the material around the STP cable, $Y^{\prime}$ is the PUL admittance of the line.

There are the effects of a lossy ground plane serving as a return conductor, so that the propagation constants

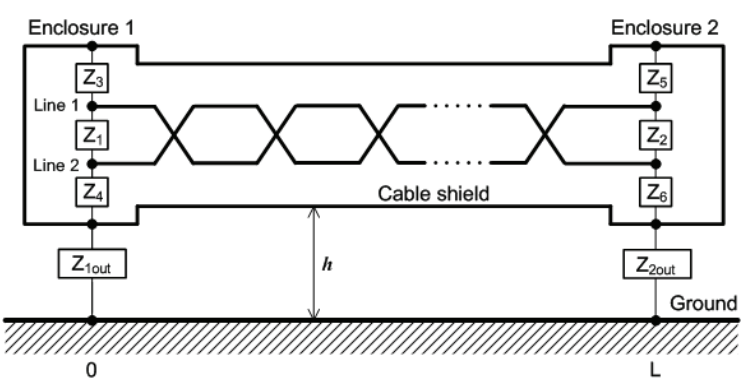

Fig. 2. Geometry of a STP cable over a ground plane

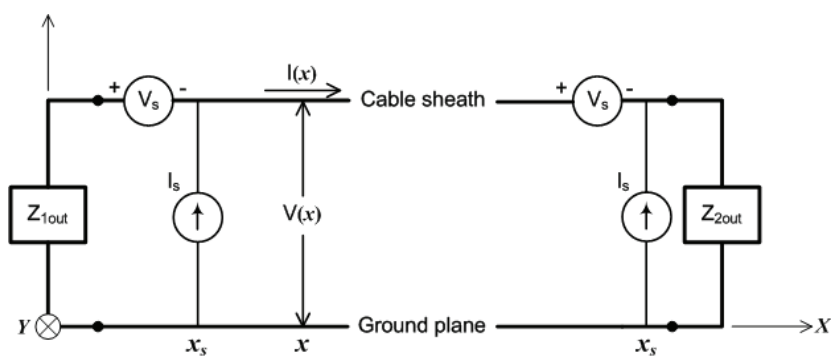

Fig. 3. Modeling of external field excitation of a STP cable as external problem. 
become complex quantities [11] and the additional impedance and admittance elements have to be considered when the PUL parameters are calculated. There are also several different expressions for the ground impedance. In this paper, we used the approximation of the ground impedance expression that is independent of the angle of incidence and very simple but accurate expression for Sunde's expression [12] given by

$$
Z_{g}=\frac{j \omega \mu}{2 \pi} \ln \frac{1+\gamma_{g} h}{\gamma_{g} h}
$$

where $\gamma_{g}$ is the wave propagation constant in the ground. As discussed by Vance [10], the ground admittance term is related to the ground impedance term $Z_{g}$ as follow

$$
Y_{g}=\frac{\gamma_{g}^{2}}{Z_{g}}
$$

Based on the definition in (3) and (4), the PUL impedance and admittance parameters are defined as

$$
\begin{gathered}
Z=j \omega L^{\prime}+Z_{g} \\
Y=\left(\frac{1}{j \omega C^{\prime}}+\frac{1}{Y_{g}}\right)^{-1}
\end{gathered}
$$

where $L^{\prime}$ and $C^{\prime}$ are, respectively, inductance and admittance terms of STP cable over the ground plane as discussed in [3]. General solutions for the voltage and current at an arbitrary point of a cable sheath can be evaluated by the sum of two cases that are influenced by a series voltage and current sources at position of the distributed sources $x_{s}$.

Then, the actual solution of voltage and current responses of any positions [1] are superposition integral of following equations. First, for $x>x_{s}$ the resulting expressions are

$$
\begin{aligned}
V(x) & =\frac{e^{-\gamma x}+\rho_{2} e^{\gamma(x-2 L)}}{2\left(1-\rho_{1} \rho_{2}\right) e^{-2 \gamma L}}\left[\left(e^{\gamma x_{s}}-\rho_{1} e^{-\gamma x_{s}}\right) V_{s}\right. \\
& \left.+\left(e^{\gamma x_{s}}+\rho_{1} e^{-\gamma x_{s}}\right) Z_{c} I_{s}\right] \\
I(x) & =\frac{e^{-\gamma x}-\rho_{2} e^{\gamma(x-2 L)}}{2 Z_{c}\left(1-\rho_{1} \rho_{2}\right) e^{-2 \gamma L}}\left[\left(e^{\gamma x_{s}}-\rho_{1} e^{-\gamma x_{s}}\right) V_{s}\right. \\
& \left.+\left(e^{\gamma x_{s}}+\rho_{1} e^{-\gamma x_{s}}\right) Z_{c} I_{s}\right]
\end{aligned}
$$

Secondly, for $x<x_{s}$ the resulting expressions are

$$
\begin{aligned}
V(x) & =\frac{e^{\gamma(x-L)}+\rho_{1} e^{-\gamma(x+L)}}{2\left(1-\rho_{1} \rho_{2}\right) e^{-2 \gamma L}}\left[-\left(e^{\gamma\left(L-x_{s}\right)}-\rho_{2} e^{-\gamma\left(L-x_{s}\right)}\right) V_{s}\right. \\
& \left.+\left(e^{\gamma\left(L-x_{s}\right)}+\rho_{2} e^{-\gamma\left(L-x_{s}\right)}\right) Z_{c} I_{s}\right]
\end{aligned}
$$

$$
\begin{aligned}
I(x) & =\frac{e^{\gamma(x-L)}+\rho_{1} e^{-\gamma(x+L)}}{2 Z_{c}\left(1-\rho_{1} \rho_{2}\right) e^{-2 \gamma L}}\left[-\left(e^{\gamma\left(L-x_{s}\right)}-\rho_{2} e^{-\gamma\left(L-x_{s}\right)}\right) V_{s}\right. \\
& \left.+\left(e^{\gamma\left(L-x_{s}\right)}+\rho_{2} e^{-\gamma\left(L-x_{s}\right)}\right) Z_{c} I_{s}\right]
\end{aligned}
$$

where $\rho_{1,2}$ are the reflection coefficients at each load and $\gamma$ is the propagation constant along the TL given by

$$
\gamma=\sqrt{Z Y}
$$

The geometrical configuration of the STP cable above ground plane is: $L=30 \mathrm{~m}, h=0.3 \mathrm{~m}, \varepsilon_{r}=10, \sigma=0.01 \mathrm{~S} / \mathrm{m}$. The characteristic impedance $Z_{c}=293 \Omega$, the termination impedance at $x=0$ and $x=L$ are set to the values $Z_{1,2 \text { out }}=\infty$. The STP cable is excited by a vertically polarized high altitude electromagnetic pulse (HEMP) [13] that is described by double exponential waveform by

$$
E_{\text {inc }}(t)=E_{0} \times\left(e^{-a t}-e^{-b t}\right)
$$

where field intensity constant $E_{0}=6.5 \times 10^{4} \mathrm{~V} / \mathrm{m}$, decay constant $a=4 \times 10^{6} \mathrm{rad} / \mathrm{s}$, rise time constant $b=6 \times 10^{8} \mathrm{rad} / \mathrm{s}$ and the incidence angle of the external HEMP is set to $\theta=$ $30^{\circ}$. The total field affecting the STP cable above the ground plane is the sum of the incident field and the reflected field from ground plane, which are expressed by [14]. Using (7) and (8), the spatial distribution of the line current and voltages can be evaluated. The load responses can be calculated by setting $x=L$.

\subsection{Internal problem}

The external EM field can give rise to disturbing currents and voltages on the internal conductor due to diffusion of EM fields through the sheath material or penetration of the fields through the small apertures of the shields. The protection capability of the STP cable is reflected by the amount of reduction of the electric field component due to the skin depth attenuation. This reduction capability will be determined precisely through the transfer impedance, which is defined as the ratio between the inner electric field and the total shield current in the frequency domain. The coupling between the external and internal TL is operated by the transfer impedance and admittances. These transfer parameters give rise to induced voltage and current sources [15]. The behavior of the induced response on the inner conductors of a STP cable are then related to the external line responses given by

$$
\begin{gathered}
V_{s}=Z_{t} \times I \\
I_{s}=-Y_{t} \times V
\end{gathered}
$$

where $Z_{t}$ and $Y_{t}$ are the transfer impedance and transfer admittance of the shield. 
For the internal problem, a modeling algorithm different from the uniform MTL theory is required, because the cross domain of the twisted pairs must be considered. It means that the distances from cable sheath to each line are changed iteratively, which can be identified by Fig. 4(a). As shown in Fig. 4(b) and (c), the whole twisted pair line has to be analyzed by separating in odd and even mode pairs. The odd mode pairs have different values of height $h_{1}, h_{2}$ in line 1 in order, and $h_{2}, h_{1}$ in line 2 in order. The proposed expanded chain matrix $C 1$ can be obtained by interacting with each chain matrix in order $\Psi_{1}, \Psi_{2}$, involved with PUL parameters taking into account iterative changed height between the line and ground plane. It can be written by

$$
\begin{aligned}
& C 1=\left[\Psi_{1}\right]\left[\Psi_{2}\right] \\
& =\left[\begin{array}{cccc}
\cosh \gamma \ell & 0 & X L_{1 g} & X L_{m} \\
0 & \cosh \gamma \ell & X L_{m} & X L_{2 g} \\
X C_{1 g} & -X C_{m} & \cosh \gamma \ell & 0 \\
-X C_{m} & X C_{2 g} & 0 & \cosh \gamma \ell
\end{array}\right] \\
& \times\left[\begin{array}{cccc}
\cosh \gamma \ell & 0 & X L_{2 g} & X L_{m} \\
0 & \cosh \gamma \ell & X L_{m} & X L_{1 g} \\
X C_{2 g} & -X C_{m} & \cosh \gamma \ell & 0 \\
-X C_{m} & X C_{1 g} & 0 & \cosh \gamma \ell
\end{array}\right] \\
& C 2=\left[\Psi_{2}\right]\left[\Psi_{1}\right] \\
& =\left[\begin{array}{cccc}
\cosh \gamma \ell & 0 & X L_{2 g} & X L_{m} \\
0 & \cosh \gamma \ell & X L_{m} & X L_{1 g} \\
X C_{2 g} & -X C_{m} & \cosh \gamma \ell & 0 \\
-X C_{m} & X C_{1 g} & 0 & \cosh \gamma \ell
\end{array}\right] \\
& \times\left[\begin{array}{cccc}
\cosh \gamma \ell & 0 & X L_{1 g} & X L_{m} \\
0 & \cosh \gamma \ell & X L_{m} & X L_{2 g} \\
X C_{1 g} & -X C_{m} & \cosh \gamma \ell & 0 \\
-X C_{m} & X C_{2 g} & 0 & \cosh \gamma \ell
\end{array}\right]
\end{aligned}
$$

where $C_{l g, 2 g}$ are the PUL capacitances induced by the electric coupling of lines 1 and $2, L_{l g, 2 g}$ are the PUL inductances induced by the magnetic coupling of ground plane to lines 1 and 2 , and $C_{m}, L_{m}$ are mutual coupling terms. These parameters can be calculated using equation presented in chapter 5 [3]. And parameter $X$ is given by $X=j \omega \ell[\sinh (\gamma \ell) / \gamma \ell]$. In contrast, the even mode pairs have an opposite structure to the odd mode pairs and it can be represented by expanded chain matrix $C 2$, as in (14).

It can be thought that the STP cable is modeled as a cascaded structures that consist of odd pair Eq. (13) and even pair equation (14) of each twisted pair as shown in Fig. 5. The expanded chain matrix relates the voltage and current at the right end of the line to the voltage and

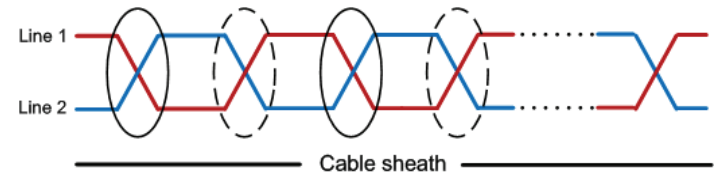

(a)

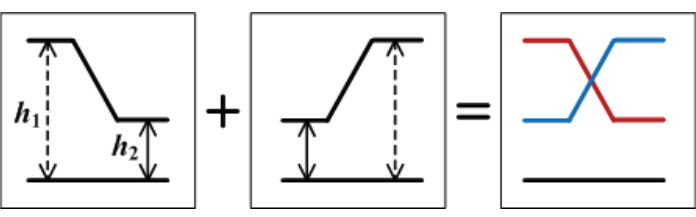

(b)

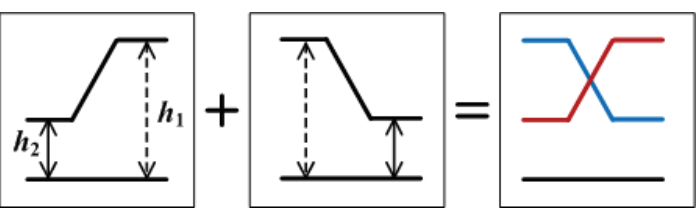

(c)

Fig. 4. Configuration of STP cable for modeling (a) Whole STP cable structure, (b) Odd number of pairs in STP cable, and (c) Even number of pairs in STP cable.
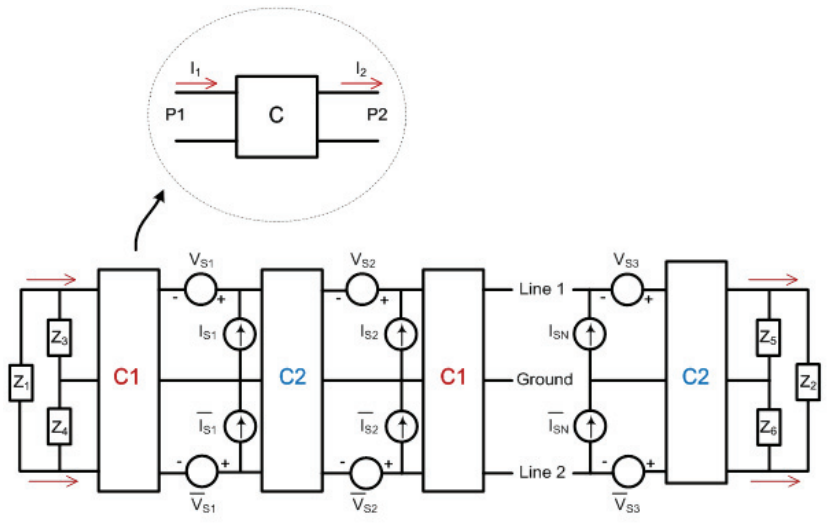

Fig. 5. Modeling of STP cable as cascaded structure for expanded chain matrix composed of odd and even pairs.

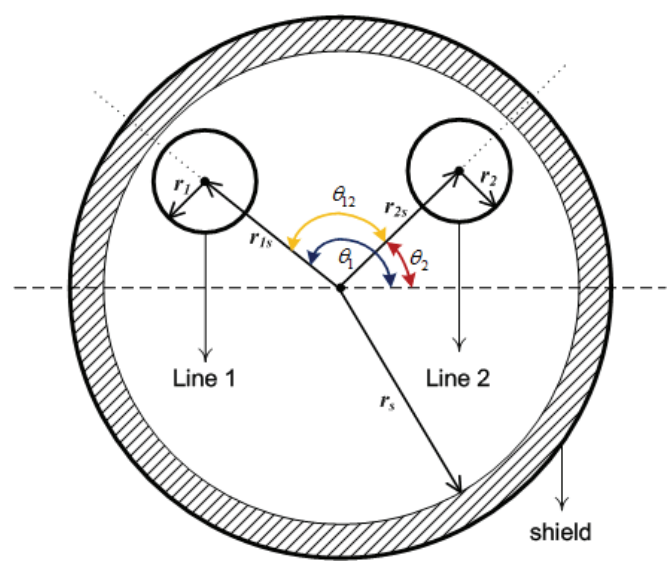

Fig. 6. Cross sectional structure of STP cable. 
current at the left end of the line. Hence, the overall expanded chain matrix of several such lines that are cascaded in series can be obtained as the product of the chain parameter matrices of the section like Fig. 5. In the proposed method, there is an important point about modeling approach used chain matrix, which means the direction of the current at port 2 is reversed from that in the definitions of the general $n$-port network systems.

Next, the cross-sectional structure of STP cable to calculate PUL parameters is shown in Fig. 6. The two lines of radii $r_{12}$ are within a shield, the interior radius of the shield is denoted by $r_{s}$ and the distances of the lines from the shield axis are denoted by $r_{l s, 2 s}$, and the angular separations are denoted by $\theta_{12}$. If the lines are widely separated from each other and the shield, it can be thought that the currents are uniformly distributed around the line and shield peripheries. Thus, the PUL inductance parameters using the wide separation approximations [3] are given by

$$
\begin{gathered}
L_{i s}=\frac{\mu}{2 \pi} \ln \left(\frac{r_{s}^{2}-r_{i s}^{2}}{r_{s} r_{i}}\right) \\
L_{m}=\frac{\mu}{2 \pi} \ln \left(\frac{r_{2 s}}{r_{s}} \sqrt{\frac{\left(r_{1 s} r_{2 s}\right)^{2}+r_{s}^{4}-2 r_{1 s} r_{2 s} r_{s}^{2} \cos \theta_{12}}{\left(r_{1 s} r_{2 s}\right)^{2}+r_{2 s}^{4}-2 r_{1 s} r_{2 s}^{3} \cos \theta_{12}}}\right)
\end{gathered}
$$

where $L_{i s}$ are PUL inductance terms of $i$-th line to shield and $L_{m}$ is mutual component.

$$
\begin{aligned}
& \frac{V_{3}}{Z_{3}}=\frac{\left(V_{4}-V_{3}\right)}{Z_{1}}-I_{3} \\
& \frac{V_{4}}{Z_{4}}=\frac{\left(V_{3}-V_{4}\right)}{Z_{1}}-I_{4} \\
& \frac{V_{5}}{Z_{5}}=\frac{\left(V_{6}-V_{5}\right)}{Z_{2}}-I_{5}
\end{aligned}
$$

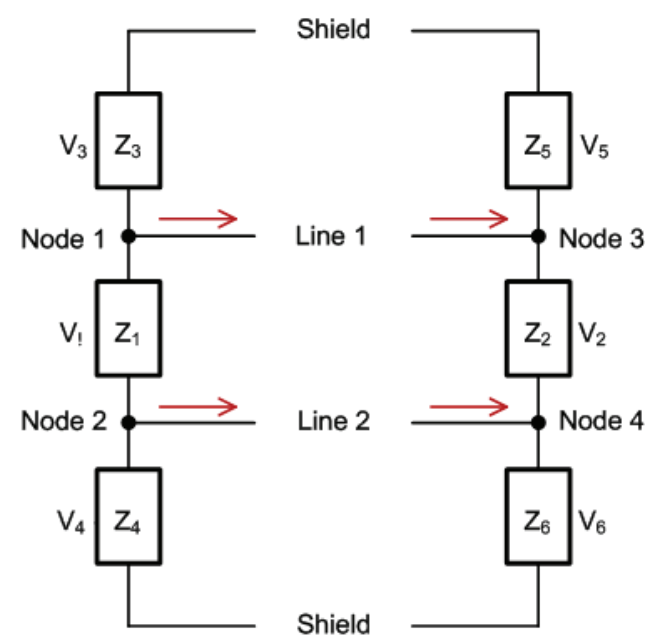

Fig. 7. Equivalent circuit for boundary conditions.

$$
\frac{V_{6}}{Z_{6}}=\frac{\left(V_{5}-V_{6}\right)}{Z_{2}}-I_{6}
$$

Fig. 7 shows an equivalent circuit for the STP cable to apply boundary conditions at each load. The node equations can be calculated from (17) to (20) using simple circuit theory. Finally, the governing equation, which is

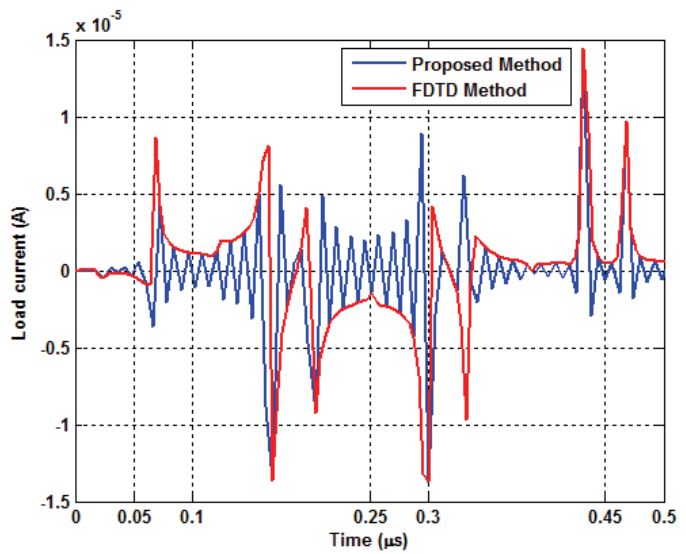

(a)

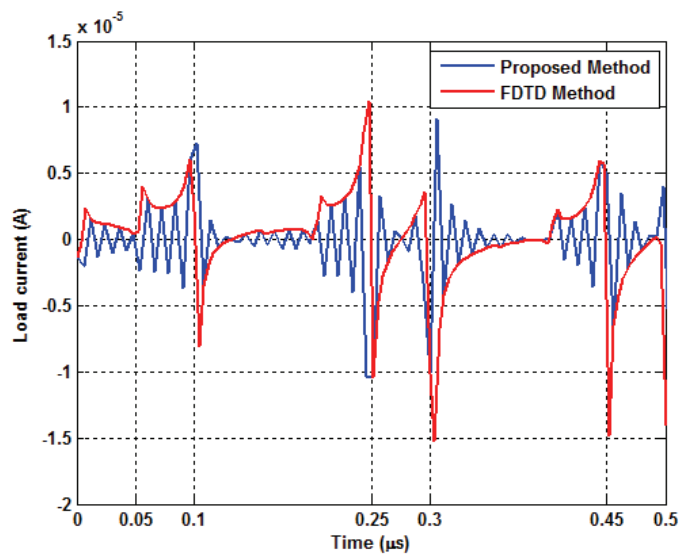

(b)

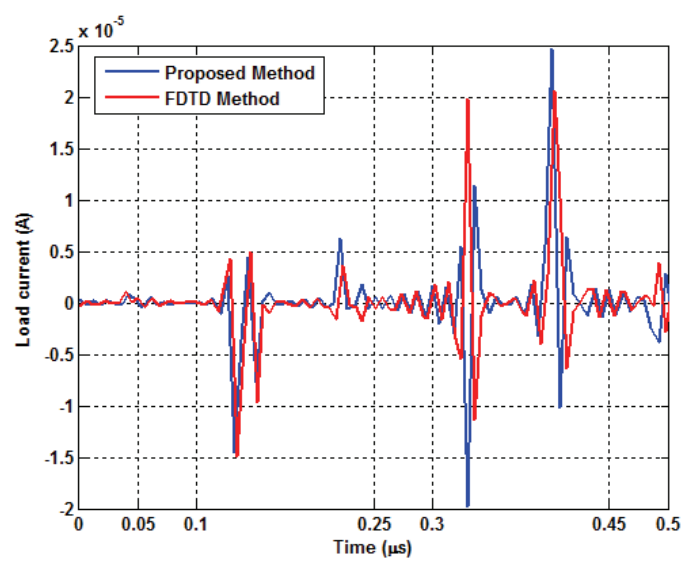

(c)

Fig. 8. Internal load current response of STP cable for different length at $h=0.3 \mathrm{~m}, \sigma=\infty$, and $Z_{1,2}=50 \Omega$. (a) $L=20 \mathrm{~m}$, (b) $L=30 \mathrm{~m}$, (c) $L=40 \mathrm{~m}$. 


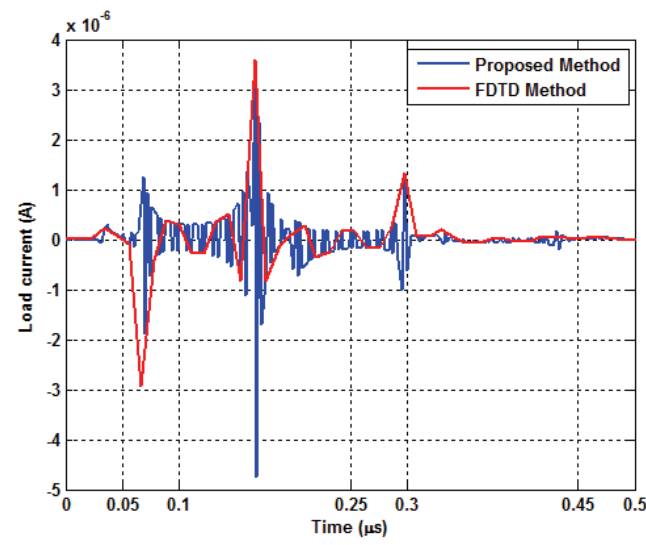

(a)

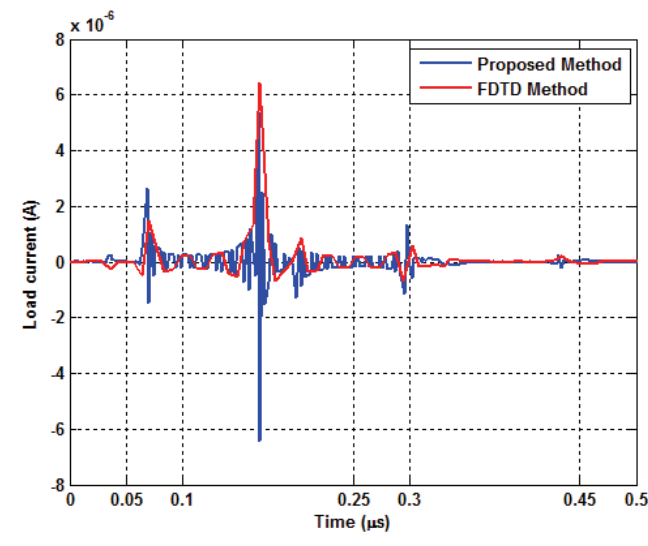

(b)

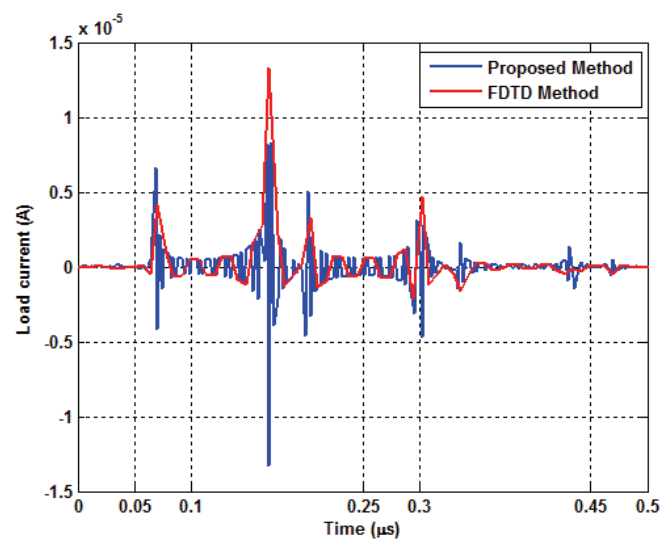

(c)

Fig. 9. Internal load current response of STP cable for different ground conductivities with $L=20 \mathrm{~m}, h=0.3$ $\mathrm{m}$, and $Z_{1,2}=50 \Omega$. (a) $\sigma=0.01 \mathrm{~S} / \mathrm{m}$, (b) $\sigma=0.1 \mathrm{~S} / \mathrm{m}$, (c) $\sigma=1 \mathrm{~S} / \mathrm{m}$.

described by multiplying the expanded chain matrices $C 1$ and $C 2$ repetitively, is given by

$$
\left[\begin{array}{l}
V_{3} \\
V_{4} \\
I_{3} \\
I_{4}
\end{array}\right]=[C 1 \cdot C 2]^{\frac{N}{2}} \times\left[\begin{array}{c}
V_{5} \\
V_{6} \\
I_{5} \\
I_{6}
\end{array}\right]+
$$

$$
+[C 1]^{\frac{N}{2}}[C 2]^{\frac{N}{2}-1} \times\left[\begin{array}{c}
-V_{S N} \\
-\bar{V}_{S N} \\
-I_{S N} \\
-\bar{I}_{S N}
\end{array}\right]+\ldots+[C 2] \times\left[\begin{array}{c}
-V_{S 1} \\
-\bar{V}_{S 1} \\
-I_{S 1} \\
-\bar{I}_{S 1}
\end{array}\right]
$$

where $N$ is the total number of twisted pairs. The expanded chain matrices of the uniform sections are multiplied together along with chain matrices that give an interchange of the voltages and currents at the junctions. So, in case of STP cable, the expanded chain matrix $[C 1 \cdot C 2]$ is the N/2-th power of the chain matrix of each section. Prior to analyzing this problem, we considered the internal line with the following parameters: transfer impedance $Z_{t}=$ $0.014 \Omega / \mathrm{m}$, transfer admittance $Y_{t}=0 \mathrm{~S} / \mathrm{m}$ [11], interior radius of the shield $r_{s}=0.15 \mathrm{~cm}$, distances of the inner line from the shield axis $r_{l s, 2 s}=0.02 \mathrm{~cm}, 0.03 \mathrm{~cm}$, each inner line radius $r_{1,2}=0.01 \mathrm{~cm}$, angle of line 1 to $2 \theta_{12}=\pi$, and termination impedances at each load in STP cable $Z_{1}=$ $Z_{2}=50 \Omega, Z_{3}=Z_{4}=Z_{5}=Z_{6}=\infty$. All of the results are from these termination impedance. We used these values in the governing equation to solve the internal problem. To solve the load responses of the STP cable, first, we have to calculate the current and voltage of shield as an external problem. These values contributed to the effect of external field coupling to STP cable and are connected to transfer parameter as shown in Fig. 1. Then, the expanded chain matrix $C 1, C 2$ are determined, the load responses can be obtained by calculating the governing Eq. (21) in frequency domain. Responses in the time domain can be more useful than the spectral responses in understanding the external field coupling mechanism to a line.

Although the Eq. (21) provides results in the frequency domain, we used the inverse Fourier transform techniques to transform a wideband spectrum into the time domain. Fig. 8 represents the induced current at load for the STP cable above ground plane for different values of the line length.

Fig. 9 shows the effect of the lossy ground plane increases the amplitude of current induced in the line. The fact that the response is smaller is due to the fact that the tangential excitation electric field along the horizontal section of the line is smaller for the case of the perfectly conducting ground. Fig. 10 shows variations of the line responses as the height of the line $\mathrm{h}$ is varied from $0.1 \mathrm{~m}$ to $1 \mathrm{~m}$. These figures indicate that increasing the height of the over the ground plane will tend to increase its induced response.

\section{Verification}

To validate the proposed method, the load responses of STP cable have been examined by the FDTD method 


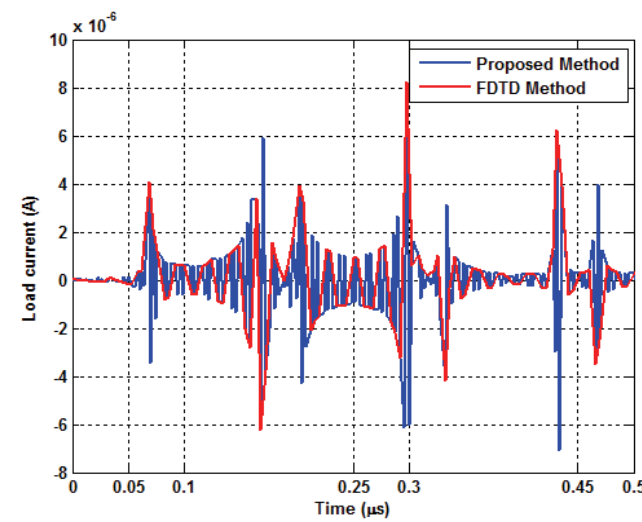

(a)

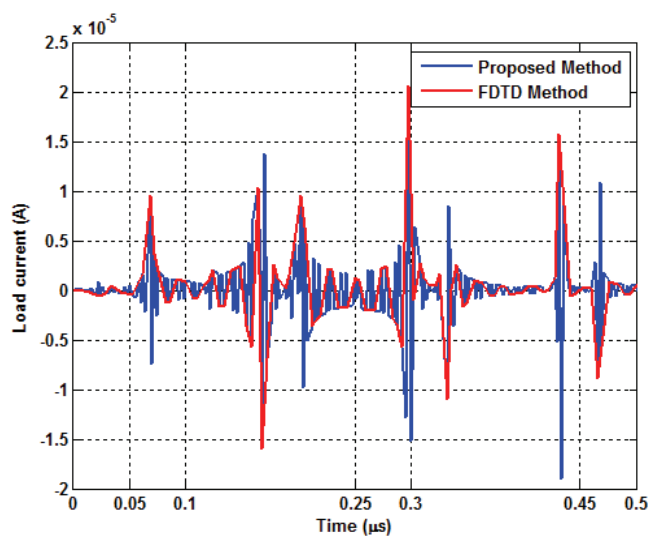

(b)

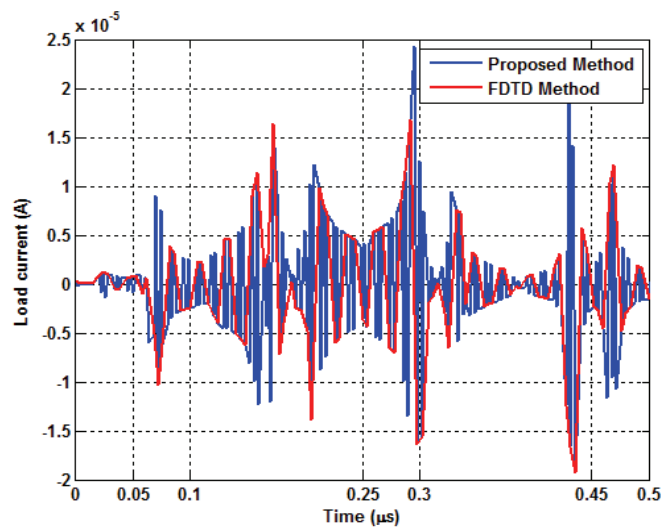

(c)

Fig. 10. Internal load current response of STP cable for different height at $L=20 \mathrm{~m}, \sigma=\infty$, and $Z_{1,2}=50$ $\Omega$. (a) $h=0.1 \mathrm{~m}$, (b) $h=0.5 \mathrm{~m}$, (c) $h=1.0 \mathrm{~m}$.

directly as 3D full wave analysis shown in Fig. 11. In the FDTD code, the excitation is vertically polarized plane wave field with an Eq. (10) as HEMP, assuming $\Delta=\lambda / 20$, $\Delta t=\Delta /(2 c)$, where $c$ is the free space velocity. A cell size has been chosen as $\lambda / 10$ at the highest frequency. The computational space is terminated with perfectly matched layer (PLM) with maximum reflection of $1 \%$. The load response of STP cable computed by FDTD method is reported in Figs. 8, 9 and Fig. 10. From this figure, it has

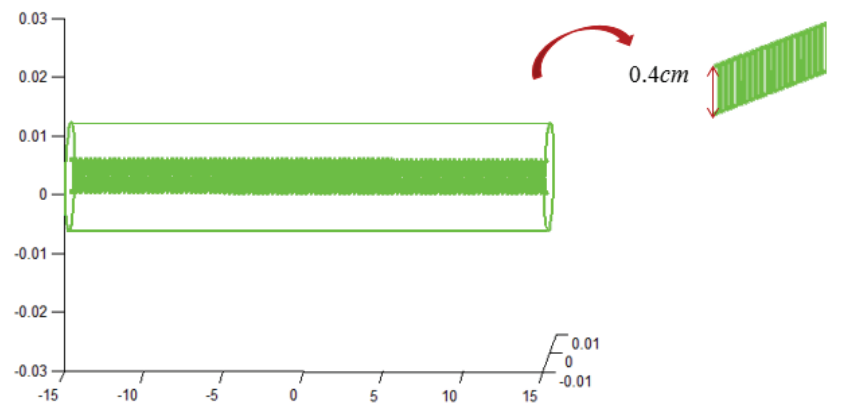

Fig. 11. A real capture of simulation.

some mismatching between proposed and reference data. The cause of these effects can be thought that the proposed method applied the approximated modeling structures which cannot contain the real model perfectly, and these are results in some error as well as numerical error. Finally, we can concluded that the result implemented by proposed method is similar with the FDTD method, which ensure the accuracy of the proposed method. Though one can conclude that the all of the structures in proposed method are modeled as approximated modeling structures, not a real case.

\section{Conclusion}

In this paper, an expanded chain matrix modeling algorithm has been proposed to analyze STP cable and verified in comparison with results from the FDTD method. Unlike the traditional modeling algorithm including numerical method for analyzing field coupling with STP cable, we applied the concept of a chain matrix composed of ABCD parameters. In the process of solving this problem, we separated the entire problem as two parts as an external and an internal problem. After the external problem was solved, by exploiting the proposed expanded chain matrix, we were able to analyze the internal response of the STP cable when the HEMP incidence occurs suddenly. This is more efficient than computing the FDTD method in terms of computer memory and computation time, listed in Table 1. It means that the proposed modeling algorithm overcomes the computation burden and limitation of the conventional approach that is applied only to the analysis of field coupling with a uniform cable line.

Table 1. Comparison of calculation time average

\begin{tabular}{c|c}
\hline Algorithm & Time (second) \\
\hline FDTD (3D Full wave analysis) & 1801.6 \\
\hline Proposed Method & 654.8 \\
\hline
\end{tabular}

\section{References}

[1] F. M. Tesche, M. V. Ianoz, and T. Karlsson, EMC Analysis Methods and Computation Models, New 
York: Wiley, 1997.

[2] M. Feliziani and F. Maradei, "Full-Wave Analysis of Shielded Cable Configurations by the FDTD Method," IEEE Trans. Magn., vol. 38, no. 2, pp. 761764, Mar. 2002.

[3] C. R. Paul, Analysis of Multiconductor Transmission Lines, John Wiley \& Sons, 1994.

[4] Frederickm. Tesche, and Paul R. Barnes, "Transient response of a distribution circuit recloser and control Unit to a High-Altitude Electromagnetic Pulse (HEMP) and lightning," IEEE Trans. Electromagn. Compat., vol. 32, no. 2, pp. 113-124, May. 1990.

[5] Y. Cho, J. Ro, Y. Chung, C. Cheon, H. Jung, "Investigation of Electromagnetic Field Coupling with Twisted Conducting Line by Expanded Chain Matrix,” JEET., vol. 8, no. 2, pp. 364-370, Mar. 2013.

[6] R. Holland and L. Simpson, "Finite-difference analysis of EMP coupling to thin struts and wires," IEEE Trans. Electromag. Compat., vol. 23, no. 2, pp. 88-97, May. 1981.

[7] K. R. Umashankar, A. Taflove, and B. Becker, "Calculation and experimental validation of induced currents on coupled wires in an arbitrary shaped cavity," IEEE Trans. Antennas Propagat., vol. 35, no. 11, pp. 1248-1257, Nov. 1987.

[8] M. Feliziani and F. Maradei, "Field-to-wire coupling using the finite element-time domain (FE-TD) method," IEEE Trans. Magn., vol. 31, pp. 1586-1589, no. 3, May. 1995.

[9] C. Liu, L. Wang, and G. Zhang, "Analysis of Transient Electromagnetic Field Coupling to Shielded Twistedpairs," 3rd International Joint Conf. Computational Science and Optimization (CSO), China, 2010, pp. 95-98.

[10] F. M. Tecshe, "An Overview of Transmission Line Analysis," Proceedings of EMC EXPO '87 International Conference on EMC, San Diego, CA, May, 1987.

[11] Vance, E. F., Coupling to Shielded Cables, R. E. Krieger, Melbourne, FL, 1987.

[12] Sunde, E. D., Earth Conduction Effects in Transmission Systems, Van Nostrand, New York, 1949.

[13] MIL-STD-188-125, "High-Altitude Electromagnetic Pulse (HEMP) Protection for Ground-Based C41 Facilities Performing Critical, Time-UR," 1990.

[14] M. Aguet, M. Ianovici and Chung-Chi Lin, "Transient Electromagnetic Field coupling to long Shielded Cables," IEEE Trans. Electromag. Compat., vol. EMC-22, pp. 276-282, No. 4, Nov. 1980.

[15] A. Orlandi, "Circuit model for bulk curr. ent injection test on shielded coaxial cables," IEEE Trans. Electromagn. Compat., vol. 45, pp. 602-615, Nov 2003.

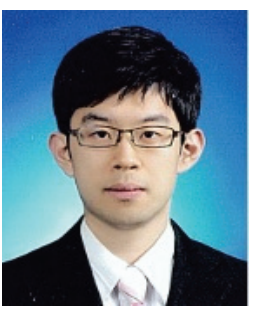

Yong-Sun Cho He received his B.S. degree in Electronic Engineering and Avionics at Korea Aerospace University in 2008. From 2008 to 2010 , he has studied for his M.S. degree in Department of Electronic Engineering at Hanyang University, Seoul, Korea. $\mathrm{He}$ is currently working towards his Ph.D. degree in Department of Electrical and Computer Engineering, Seoul National University of Korea. His research interests are numerical analysis of microwave, Electromagnetic Compatibility (EMC/EMI). He is also interested in signal processing for image reconstruction using RADAR system and inverse scattering problem using optimization methods.

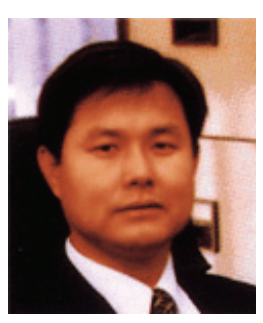

Hyun-Kyo Jung He received his B.S., M.S., and Ph.D. degrees in electrical engineering from Seoul National University, Seoul, Korea, in 1979, 1981, and 1984, respectively. From 1985 to 1994, he was a member of the faculty at Kangwon National University. From 1987 to 1989 , he was with the Polytechnic University of Brooklyn, Brooklyn, NY. From 1999 to 2000, he was a Visiting Professor with the University of California at Berkeley. He is currently a Professor of the Department of Electrical and Computer Engineering, Seoul National University, Seoul, Korea. His research interests are various fields of the analysis and the design of electric machines and the numerical analysis of microwave, HPEM, EMI/EMC and electrical systems, especially with the FEM.

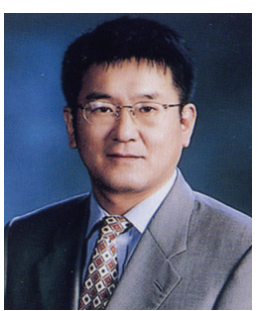

Changyul Cheon He received his B.S. degree and the M.S. degree in electrical engineering from the Seoul National University, Seoul, Korea in 1983 and 1985, respectively, and the Ph.D. degree in electrical engineering from the University of Michigan at Ann Arbor in 1992. From 1992 to 1995 , he was with the Department of Electrical Engineering, Kangwon National University, Chuncheon, Korea, as an Assistant Professor. $\mathrm{He}$ is currently Professor of Electrical Engineering at the University of Seoul (UoS), Seoul, Korea. His group at UoS is currently involved with the design and analysis of microwave and millimeter-wave passive device using FEM, FDTD, and MoM techniques as well as analysis of scattering problem. He is also interested in microwave application for biological applications. 


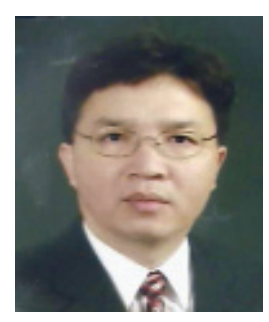

Young-Seek Chung $\mathrm{He}$ received his B.S., M.S., and Ph.D. degrees in electrical engineering from Seoul National University, Seoul, Korea, in 1989, 1991, and 2000, respectively. From 1991 to 1996, he was with the Living System Laboratory, LG Electronics. From 1998 to 2000, he was a Teaching Assistant of electrical engineering with Seoul National University. From 2001 to 2002, he was with Syracuse University, Syracuse, NY. From 2003 to 2005, he has been a faculty member with the Department of Communication Engineering, Myongji University, Kyunggi, Korea. He is currently a Professor of the Department of Electronics Convergence Engineering, Kwangwoon University, Seoul, Korea. His current interests are numerical analysis and inverse scattering using time-domain techniques. 